\title{
Four new loci associations discovered by pathway-based and network analyses of the genome-wide variability profile of Hirschsprung's disease
}

\author{
Raquel Ma Fernández ${ }^{1,2}$, Marta Bleda 2,3, Rocío Núñez-Torres ${ }^{1,2}$, Ignacio Medina ${ }^{3,4}$, Berta Luzón-Toro 1,2, \\ Luz García-Alonso3, Ana Torroglosa 1,2, Martina Marbà3,4, Ma Valle Enguix-Riego 1,2, David Montaner ${ }^{3}$, \\ Guillermo Antiñolo ${ }^{1,2}$, Joaquín Dopazo ${ }^{2,3,4^{*}}$ and Salud Borrego ${ }^{1,2^{*}}$
}

\begin{abstract}
Finding gene associations in rare diseases is frequently hampered by the reduced numbers of patients accessible. Conventional gene-based association tests rely on the availability of large cohorts, which constitutes a serious limitation for its application in this scenario. To overcome this problem we have used here a combined strategy in which a pathway-based analysis (PBA) has been initially conducted to prioritize candidate genes in a Spanish cohort of 53 trios of short-segment Hirschsprung's disease. Candidate genes have been further validated in an independent population of 106 trios. The study revealed a strong association of 11 gene ontology (GO) modules related to signal transduction and its regulation, enteric nervous system (ENS) formation and other HSCR-related processes. Among the preselected candidates, a total of 4 loci, RASGEF1A, IQGAP2, DLC1 and CHRNA7, related to signal transduction and migration processes, were found to be significantly associated to HSCR. Network analysis also confirms their involvement in the network of already known disease genes. This approach, based on the study of functionally-related gene sets, requires of lower sample sizes and opens new opportunities for the study of rare diseases.
\end{abstract}

Keywords: HSCR, Pathway-based analysis, Network analysis, GWAS

\section{Background}

Understanding complex genetic diseases is one of the great challenges for $\mathrm{XXI}^{\mathrm{st}}$ century medicine. In the past few years, the development of new tools, such as Genome-Wide Association Studies (GWAS) and Next Generation Sequencing (NGS), has allowed the identification of hundreds of genetic variants associated to different diseases that provide new insights of their genetic architecture. Hirschsprung's disease (HSCR, OMIM 142623) is a nice example of the current situation of many of the

\footnotetext{
* Correspondence: jdopazo@cipf.es; salud.borrego.sspa@juntadeandalucia.es ${ }^{2}$ Centre for Biomedical Network Research on Rare Diseases (CIBERER), Barcelona, Spain

'Department of Genetics, Reproduction and Fetal Medicine, Institute of Biomedicine of Seville (IBIS, University Hospital Virgen del Rocío/CSIC/ University of Seville, Seville, Spain

Full list of author information is available at the end of the article
}

complex genetic diseases. HSCR or aganglionic megacolon is a rare developmental disorder of variable penetrance and expressivity, male predominance, and an incidence of $1 / 5000$ newborn human infants. It is a neurocrystopathy characterized by the absence of intramural ganglion cells in the myenteric and submucosal plexuses along a variable portion of the distal intestine. The most widely accepted etiopathogenetic hypothesis for HSCR is based on a defect of craniocaudal migration of neuroblasts originating from the neural crest that, under normal circumstances, reach the small intestine in the 7th week of gestation and the rectum in the 12th week [1].

The HSCR clinical forms with variable extension of the aganglionic segment could be interpreted as interruptions of the migration process in different gestational periods: the earlier the migration arrest, the longer the distal aganglionic intestinal portion. Based on the length of the aganglionic region, the disorder is classified into

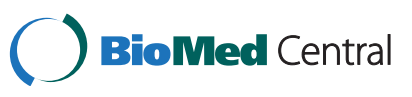


short segment (S-HSCR: aganglionosis up to the upper sigmoid colon, $80 \%$ of cases), long-segment (L-HSCR: aganglionosis up to the splenic flexure and beyond, 17\% of cases) and total colonic aganglionosis forms (TCA, $3 \%$ of cases). Significantly, HSCR displays a highly variable phenotype with variation in recurrence risk by gender, familiarity, segment length of aganglionosis and associated phenotypes [2]. The reason for most of this variation is largely unknown, although gene discovery has clarified some genotype-phenotype correlations. Numerous molecular genetic studies have identified rare coding mutations in many genes (RET, GDNF, NRTN, PSPN, GFRA1, EDNRB, EDN3, ECE1, NTF3, NTRK3, SOX10, PHOX2B, L1CAM, ZFHX1B, KIAA1279, TCF4, PROK1, PROKR1, PROKR2, NRG1, SEMA3A and SEMA3D) related to HSCR [1-8]. Moreover, several additional HSCR-associated regions, such as 9q31 [9], 3p21 [10,11], 19q12 [10], 16q23 [12], 21q21 [13] or 4q31.3q32.3 [14] have been described, although the genes underlying such associations have not been identified yet. However, cumulatively, the conventional mutations related to HSCR reported so far explain around $5 \%$ of cases, being the vast majority of them L-HSCR/TCA and syndromic forms of the disease [1,2]. Additional phenotypic variation is explained by common lowpenetrance polymorphic variants at $R E T$ (10q11.21) [2,15,16] and NRG1 [17], but the vast majority of HSCR heritability is still unknown. In other words, all these genes and loci seem to contribute to HSCR phenotype, but associations change according to the population studied and the way of contribution of the different elements is still unclear.

Conventional GWAS, based on single markers, require of large cohorts. This represents an especially remarkable limitation for the case of rare diseases, where typically a limited number of patients are available. In an attempt to overcome this limitation, pathway-based analysis (PBA) [18] has been applied to select groups of functionally-related genes collectively associated to the disease (through their corresponding SNPs) in a first analysis step. Actually, PBA has already been successfully applied to the study of several diseases [19-21]. The application of PBA tests produced a reduced list of candidate genes which was further validated with an independent set of SNPs in a larger population of 106 trios. The result of this validation was the identification of four new loci associated to the disease, RASGEF1A, IQGAP2, DLC1 and CHRNA7, accounting for different defects in biological processes related to HSCR, such as signal transduction (particularly the Ras pathway) and ENS formation. In addition, network analysis was used as supplementary evidence to support the results found. Network analysis, exploits the information contained in the interactome with the idea that proteins close in the interaction network will have a higher probability of causing the same disease and constitutes a powerful technique to detect gene-disease associations [22-24]. Network analysis has been successfully applied to discover genes in different diseases, such as ataxias [25], Huntington disease [26], schizophrenia [27] or Alzheimer's disease [28].

\section{Material and methods}

\section{Subjects}

We have included in this study a total of 159 sporadic isolated HSCR trios, composed by the affected patients (127 males, 32 females) and their unaffected parents, all of them originating from Spain. A total of 103 out of the 159 patients were S-HSCR, 31 presented with L-HSCR and the length of aganglionosis was not available for the remaining 25 cases.

For the first part of this study, consisting in the genome wide genotyping study, we selected 53 S-HSCR trios. For further validation studies of the candidate genes resulting from GWAS, we genotyped an independent selection of SNPs in an independent series of 106 trios.

An informed consent was obtained from all the participants for clinical and molecular genetic studies. The study conformed to the tenets of the declaration of Helsinki as well as the requirements established by our Institutional Review Board.

\section{Genome-wide genotyping}

Genome wide genotyping was carried out using the Affymetrix $500 \mathrm{k}$ chip (composed of the $250 \mathrm{k} \mathrm{Nsp}$ and the $250 \mathrm{k}$ Sty chips) and the Genechip ${ }^{\circledR}$ System (Affymetrix). Quality controls were as follows: SNPs missing in more than $20 \%$ of the samples in the calling process were discarded. SNPs with MAF $<0.5 \%$, with Mendelian errors or not in Hardy-Weinberg equilibrium (in unaffected samples; $\mathrm{p}$-value $<10^{-5}$ ) were also discarded. Samples with more than 5\% of the SNPs missing were not considered for further analysis. Data are available in the Gene Expression Omnibus (GEO) database (http:// ncbi.nlm.nih.gov/geo/) under the identifier GSE33732.

\section{Conventional gene association analysis in trios}

Transmission Disequilibrium Test (TDT) association statistics was carried out as implemented in the PLINK [29] software for the 53 trios included in the genome wide genotyping.

\section{Pathway-based analysis}

The SNPs were ranked according to their p-values obtained in the TDT test and a PBA test [30], as implemented in the GESBAP [31] module of the Babelomics software [32,33], was conducted. Briefly, PBA tests make 
only use of SNPs mapping within genes or in the neighborhood (here defined as 500 bps up-and downstream of the gene limits). When multiple SNPs map onto the same gene, the SNP with lowest (most significant) $\mathrm{p}$-value is retained. In this way, a list of genes ranked by the p-values of the SNPs mapping onto them is constructed. Then the PBA test analyzes the distribution of sets of functionally related genes across the list. Here we use Gene Ontology (GO) [34] terms to define the functional modules tested. GO terms significantly associated to low p-values are found upon the application of a logistic regression. To avoid false positives due to multiple-testing effects, only GO terms with a FDRadjusted [35] $\mathrm{p}$-value $<0.05$ are declared significant.

\section{PBA-based two stage study}

A widely used strategy involves the genotyping of a number of samples for a large number of markers on a first stage (discovery step), followed by a second genotyping step of a smaller selection of markers in another (larger if possible) subset of samples (validation step). The standard approach considers stage 2 as a replication study and focus on markers statistically significant when stage 2 is considered alone [36].

Here, instead of using a conventional approach based on single marker associations for the discovery step, we have followed a conceptually different approach. Our approach is inspired in an idea that is gaining popularity, that a disease is rarely the consequence of a defect in a single gene, but rather reflects the perturbations of the complex network of functionally related gene modules [23,37,38]. Therefore, we have used PBA to discover gene modules (represented by GO terms) significantly associated to the disease. Then, we consider that genes belonging to such GO modules define a subset of candidate genes to be tested in the second stage. A similar idea has already successfully been used for gene expression analysis [39].

According to the PBA carried out in the discovery stage, a total of 68 candidate genes belonging to the $\mathrm{GO}$ modules most associated to the disease were selected because of their lower p-values. A total of 190 new SNPs mapping onto the 68 candidate genes (an average of $1-3$ $\mathrm{SNP} /$ gene) were subsequently selected for further evaluation. Selection of SNPs within those genes followed 4 main criteria: they were not included in the chip used for the first genotyping study, they should be homogeneously distributed along the entire sequence of the gene (including regions susceptible of being regulatory elements), their reported allelic frequency should be of at least 5\% in Caucasian population and should have been identified as Tag SNPs by the HapMap Project. The software PupaSuite [40] was used to help in this section. Largescale validation genotyping of each SNP was performed in the independent series of 106 trios by Taqman technology using 7900HT Fast Real-Time PCR System (Applied Biosystems, Foster City, California, USA) as previously described. As usual, significance in the transmission of the alleles in the families is tested by an $X^{2}$ test, applying a Yates correction (SPSS v.17.0).

One limitation of this approach is that SNPs not mapping within (or close) to genomic elements with a functional annotation will be missed. However, the power for detection of associations of SNPs related to genes with some known function, interaction, etc., is greatly increased by this approach.

\section{Expression analyses}

Human postnatal gut specimens were obtained from endoscopic gut samples biopsied from patients in the University Hospital Virgen del Rocío because of different medical indications. Except for the case of RASGEF1A, whose expression had already been verified in ENS [16], these samples were used to verify gene expression of the genes that resulted associated to HSCR, by both RTPCR and immunohistochemistry analyses.

The cDNA specimens from those tissue samples were obtained with the gentleMACS ${ }^{\mathrm{TM}}$ Dissociator and thermoMACS ${ }^{\mathrm{TM}}$ Separator systems (Miltenyi Biotech) following the protocols recommended by the manufacturers. RT-PCR analyses were performed on such cDNA samples to test expression of each of the associated genes. As positive controls we used Brain Human Normal cDNA, Kidney Human Normal cDNA and Liver Human Normal cDNA (Invitrogen). Primer sequences and conditions are available on request.

Paraffin gut tissue sections (normal and HSCR gut, pancreas, cerebral cortex, testis and lung; $5 \mu \mathrm{m}$ thick) were dewaxed in xylene and rehydrated in a series of graded alcohols. Endogenous peroxidase activity was blocked with water containing $3 \% \mathrm{H}_{2} \mathrm{O}_{2}$ for 30 minutes. Antigen retrieval was done by microwaving using citrate phosphate buffer ( $\mathrm{pH}$ 6.0). Sections were incubated at $4{ }^{\circ} \mathrm{C}$ overnight with the primary antibodies: IQGAP2 (1:10.000 dilution, mouse monoclonal, Santa Cruz Biotechnology), CHRNA7 (1:5000 dilution, rabbit polyclonal, Sigma Aldrich) and DLC1 (1:2000 dilution, rabbit polyclonal, Sigma Aldrich). After several washes in Tris buffer, peroxidase-labeled secondary antibodies and 3,3'diaminobenzidine were applied to develop immunoreactivity, according to manufacturer's protocol (EnVision; Dako, Glostrup, Denmark). The slides were then counterstained with hematoxylin and mounted in DPX (BDH Laboratories, Poole, UK). Sections of cerebral cortex and lung were used as negative controls, while sections of pancreas and testis were used as positive controls to test the three primary antibodies. Photographs were taken at $20 \mathrm{um}$ and $50 \mathrm{um}$ the microscope BX61 (Olympus) with a digital camera DP72 and captured through the 
CellSens software. Tissues to test the antibodies (all tissues except gut) were selected according the webpage: http://www.proteinatlas.org/.

\section{Network analysis}

The information on protein interactions that define the so called interactome provides an independent view on the biological consistence of the relationship of a set of genes with a disease $[41,42]$. Here, we have studied the connectivity of the genes discovered in our analysis to the genes already known to be associated to HSCR by means of the program SNOW [43], implemented in the Babelomics package [33]. SNOW detects the largest sub-network linking all these genes and tests if some network parameters are significantly beyond their corresponding random expectations. An extended version of the interactome that includes protein interactions described in Reactome has been used here [44].

An empirical distribution of the random expectation of the parameters of a network of $\mathrm{N}$ components can be obtained by repeatedly sampling random sets of $\mathrm{N}$ genes from the complete genome and calculating the average parameters of their corresponding minimum connecting trees. Thus, the parameters obtained for the network to be tested can be contrasted with respect to their corresponding random expectations [43].

\section{Results}

\section{Pathway-based analysis}

The whole workflow-type overview of the steps carried out in this project is reflected in the Additional file 1: Figure S1. Only the RET gene was found as significantly associated to HSCR (FDR-adjusted p-value $=7.39 \times 10^{-3}$ ) upon the application of a conventional SNP-based TDT on the data resulting from the GWAS in the 53 HSCR trios. Figure 1 contains a graphical representation the distribution of p-values obtained from the TDT.

Then, we conducted the PBA test, as implemented in the Babelomics package, over the list of markers ranked by p-value (see Materials and Methods). In this discovery step, we found a total of $11 \mathrm{GO}$ modules significantly associated to HSCR, with FDR-adjusted p-values $<0.01$. Five of them were related to signal transduction and its regulation: Ras protein signal transduction (GO:0007265), regulation of Ras protein signal transduction (GO:0046578), regulation of small GTPase mediated signal (GO:0051056), regulation of Rho protein signal transduction (GO:0035023) and small GTPase mediated signal transduction (GO:0007264). With lower significance, although still significant at FDR-adjusted p-values $<0.05$, and belonging to the same GO branch and descendants of the term signal transduction, the GO terms enzyme linked receptor protein signaling pathway (GO:0007167) and regulation of signal transduction (GO:0009966) can also be considered as associated to the disease (see Table 1 and Figure 2). The Ras pathway is a well known intracellular signalling pathway, mediated by the RET receptor and involved in cell survival and proliferation. Both processes are essential in ENS formation [45]. In addition, previous studies have demonstrated that signalling through the small Rho GTPases is also important for colonization of the gut by enteric neural crest cells (ENCC) and the concomitant growth of axons [45]. These results strongly suggest that members of the Ras/Rho protein signal transduction or regulators are most probably playing a key role in the pathogenesis of HSCR, as particularly supported by the association of the GO modules above mentioned, descendant in the GO hierarchy of the small GTPase mediated signal transduction term (Figure 2).

On the other hand, migration of ENCC in the gut wall during embryogenesis requires interactions between the migrating neural crest cells and the extracellular matrix environment in different regions of the developing gut. Therefore, it results evident the key role of cell-cell adhesion (GO:0016337), with a marginal, but still significant (FDR-adjusted p-value $<0.05$ ) association to the disease, during ENS system formation. It is not surprising that genes belonging to this functional module are involved in HSCR. Our analysis also reveals other

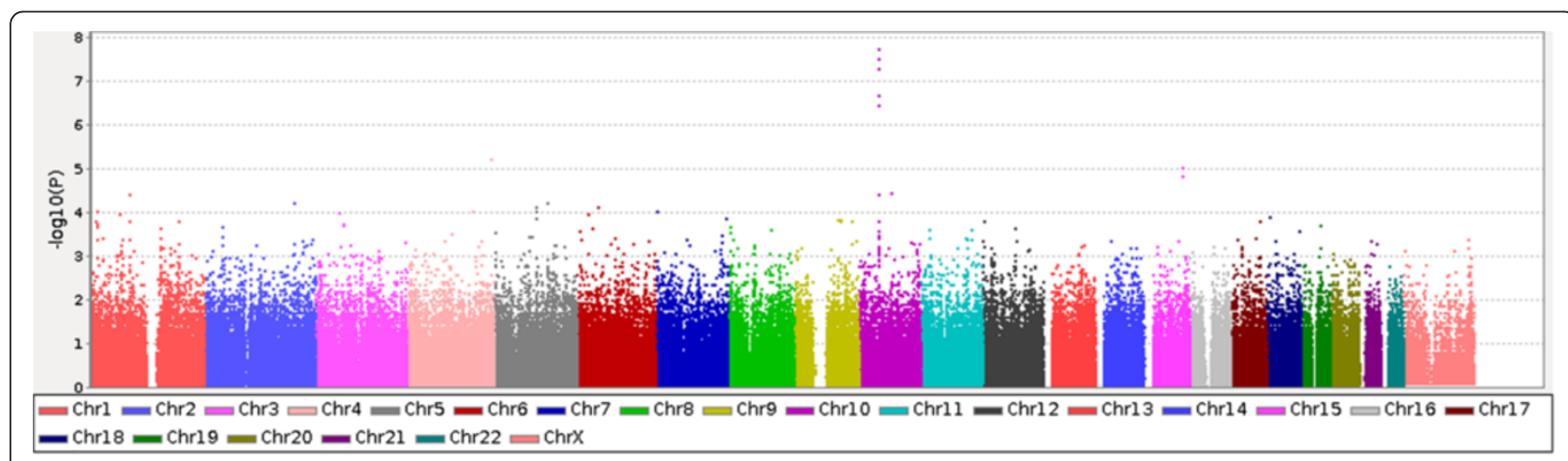

Figure 1 Graphical summary of the genome wide TDT on the first population of 53 trios used as stage 1 association. The $x$ axis represents the chromosome position in the consecutive, color-coded chromosomes and the y axis shows - $\log _{10}[p$-value]. See details in the text. 
Table $1 \mathrm{GO}$ modules significantly associated to HSCR (FDR-adjusted p-values < 0.01 )

\begin{tabular}{lll}
\hline GO ID & Definition & $\begin{array}{c}\text { Significance } \\
\text { (Adj. } \mathbf{p} \text {-val }<\mathbf{0 . 0 5} \text { ) }\end{array}$ \\
\hline GO:0051056 & regulation of small GTPase mediated signal transduction & $3.216 \times 10^{-5}$ \\
GO:0046578 & regulation of Ras protein signal transduction & $6.825 \times 10^{-5}$ \\
GO:0007268 & synaptic transmission & $4.259 \times 10^{-4}$ \\
GO:0007265 & Ras protein signal transduction & $8.245 \times 10^{-4}$ \\
GO:0007264 & small GTPase mediated signal transduction & $3.136 \times 10^{-3}$ \\
GO:0035023 & regulation of Rho protein signal transduction & $5.369 \times 10^{-3}$ \\
GO:0006816 & calcium ion transport & $6.978 \times 10^{-3}$ \\
GO:0016337 & cell-cell adhesion & 0.01203 \\
GO:0006812 & cation transport & 0.02391 \\
GO:0007167 & enzyme linked receptor protein signaling pathway & 0.03174 \\
G0:0009966 & regulation of signal transduction & 0.04825
\end{tabular}

Values in italics are marginally significant at FDR-adjusted $p$-values $<0.05$.

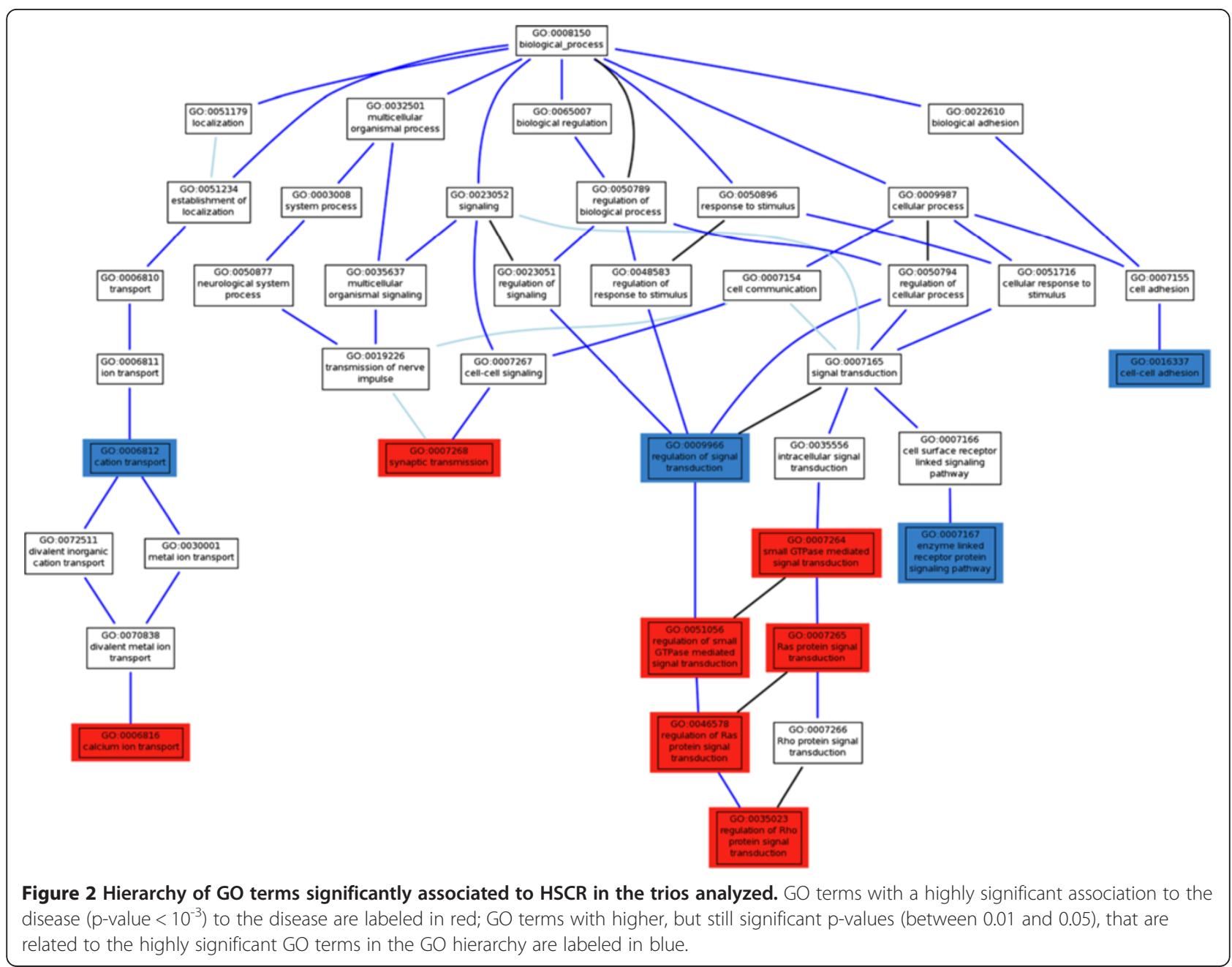


functional modules associated to the disease, such as calcium ion transport (GO:0006816) and, marginally, cation transport (GO:0006812), whose biological significance in the context of ENS formation is evidenced by the key role of ionic transport processes in cell migration context. A migrating cell has to govern cell volume regulatory ion transport mechanisms in order to create the appropriate micro- or even nano-environment in the intra- and/or extracellular space, which is necessary to guarantee the correct polarity and hence direction of movement of a migrating cell. Therefore, these associated GOs might provide potential candidate genes to be involved in ENS formation and also the pathogenesis of HSCR.

\section{Validation of candidate genes}

As previously mentioned, a conventional marker-based test rendered only a gene association to the disease, which was, as expected, the RET proto-oncogene. Excluding this gene, whose association with the disease has been already extensively reported, we selected a series of 68 additional genes represented in those HSCRassociated GO modules, in order to validate and analyze in more depth the previous functional association results. These 68 genes were those with highest nominal p-values in SNPs mapping onto them. Among those 68 genes, a selection of 190 SNPs was also done (2-3 per gene) and we tested their distribution in the whole series of trios (Additional file 2: Table S1). Genotyping of the selected variants was performed by Taqman technology and their distribution was compared between transmitted and untransmitted alleles from unaffected parents to their affected offspring. This analysis revealed a significant association of 4 out of the 68 genes analyzed, as shown in Table 2. Noteworthy, 2 genes, DLC1 and RASGEF1A, presented association to HSCR with 2 and 3 SNPs respectively, while for the remaining genes only 1 of the tested SNPs resulted in statistical significance. A detailed inspection of the associated genes in the context of the GO modules, showed that 3 of them are part of the signal transduction related terms (RASGEF1A, IQGAP2, DLC1) and the other one is part of GOs related to migration processes (CHRNA7) (Table 2).

In addition, the expression of the 4 genes in human gut specimens was verified by both immunohistochemical (Figure 3) and RT-PCR (Figure 4) analyses.

\section{Confirmatory network analysis}

In order to find additional independent evidences on the biological reliability of the genes validated, we have applied network analysis [41] to look for significant networks that link the discovered genes to already known disease genes. Thus, we applied the network significance test implemented in the SNOW program, which is now part of the Babelomics suite, to the 4 discovered genes and the genes already known to be related to HSCR by numerous studies [1-8]. Figure 5 shows a network that links a group of 12 disease genes and 3 of the 4 validated. This is a considerable number of genes participating in the network even though that there is only information on protein interactions for 11 out of the genes previously related to HSCR, and for 3 out of 4 new genes here identified. This network displays a betweenness value which is significantly higher that the random expectation $(\mathrm{p}$-value $=0.01)$. This parameter accounts for the number of shortest pathways that connect any two proteins in the sub-network passing through a given node and is an average value. Despite the biological interpretation of network parameters is not always obvious, a high betweenness is characteristic of signaling pathways, which is consistent with the findings based on the GO terms. Both types of evidences point towards processes related to signaling associated to HSCR.

Obviously only genes for which information on the interaction of their gene products is available were used in the derivation of the network. This means that the networks found are most probably an underestimation of the real network associated to the disease.

\section{Discussion}

High-throughput genomic technologies are revolutionizing biology and medicine by providing more and more resolution in the data produced. However, at the same time, are posing new challenges in the way such data can be analyzed and interpreted. In particular, conventional marker-based analysis of GWAS requires of large sample sizes to find significant associations given that some genes may be really associated with the disease, but may not reach a stringent genome-wide significance threshold required in a massive multiple testing scenario [18]. This makes of GWAS a methodology especially impracticable in the field of rare diseases. In order to overcome the limitations of conventional single-marker based association analysis, alternative approaches for the analysis of GWAS analysis have been proposed in the last few years. Beyond testing solutions that use multiple markers or marker information, such as linkage, etc., the recent proposal of PBA approaches has introduced a new angle in the analysis of GWAS data, closer to the principles of systems biology. Typically, PBA approaches check whether some statistics have consistent yet moderate deviation from chance for a group of related genes (for example, belonging to a GO category). The rationale for this approach is the accepted notion that genes do not work in an isolated way, but rather in complex molecular networks and cellular pathways that are often involved in disease susceptibility and 
Table 2 Validation of the genes in the stage II, independent population of 106 trios

\begin{tabular}{|c|c|c|c|c|c|}
\hline Gene & SNP & Allele & Transmited & Untransmited & Statistic and $p$-value \\
\hline \multirow[t]{6}{*}{ RASGEF1A (rs1254964 $\left.p=3.856 \times 10^{-05}\right)$} & rs1254958 & $G$ & $143(67.45 \%)$ & $187(88.2 \%)$ & $x^{2}=25.27 ; p=5 \times 10^{-7}$ \\
\hline & & A & 69 (35.55\%) & $25(11.8 \%)$ & \\
\hline & rs10793422 & G & $141(66.5 \%)$ & $184(86.8 \%)$ & $x^{2}=23.25 ; p=1.4 \times 10^{-6}$ \\
\hline & & A & $71(33.5 \%)$ & $28(13.2 \%)$ & \\
\hline & rs2503846 & $\mathrm{T}$ & $32(15.0 \%)$ & $71(33.5 \%)$ & $x^{2}=18.52 ; p=1.68 \times 10^{-5}$ \\
\hline & & G & $180(85.0 \%)$ & $141(66.5 \%)$ & \\
\hline \multirow[t]{2}{*}{ IQGAP2 (rs950643 $p=0.0003585)$} & rs3797412 & A & $146(68.8 \%)$ & $179(84.4 \%)$ & $x^{2}=13.49 ; p=2.393 \times 10^{-4}$ \\
\hline & & G & $66(31.2 \%)$ & $33(15.6 \%)$ & \\
\hline \multirow[t]{4}{*}{ DLC1 (rs1454947p $p=0.007526)$} & rs9325866 & $\mathrm{T}$ & $34(16.0 \%)$ & $52(24.5 \%)$ & $x^{2}=4.22 ; p=0.0401$ \\
\hline & & C & $178(8.04 \%)$ & $160(75.5 \%)$ & \\
\hline & rs536147 & $\mathrm{T}$ & $36(16.9 \%)$ & $56(24.5 \%)$ & $x^{2}=5.00 ; p=0.0252$ \\
\hline & & C & $176(83.1 \%)$ & $156(75.5 \%)$ & \\
\hline \multirow[t]{2}{*}{ CHRNA7 (rs2175886 $p=0.000607)$} & rs868437 & C & $47(22.2 \%)$ & $76(35.8 \%)$ & $x^{2}=8.98 ; p=0.0027$ \\
\hline & & $\mathrm{T}$ & 165 (77.8\%) & $136(64.2 \%)$ & \\
\hline
\end{tabular}

Genes are shown along with the best scoring SNPs mapping onto them and the corresponding p-values obtained in the stage I. See text for details.

progression $[23,41,46,47]$. Then, the use of prior biological knowledge about relationships among genes and pathways will increase the possibility of identifying the genes and mechanisms that are involved in disease pathogenesis [48]. Here we have adopted a widely accepted definition of gene functionality, which is represented by the GO. One obvious limitation of this approach is that variants not mapping within, or close to, genomic elements with a functional annotation will be missed. However, it is known that coding genes and functional elements in their neighborhood (e.g. splice acceptor and donor sites, transcription factor binding sites, etc.) harbor $85 \%$ of the mutations with large disease related effects $[49,50]$.

In this study we have applied a new approach which can be considered a functional version of a two stage analysis of GWAS data. In our approach, the conventional discovery stage based on markers is substituted by a PBA, that allows us to identify biological processes (represented by GO terms) associated to the disease in the Spanish population. The subsequent step of validation allowed us to identify 4 new gene associations to the disease among the ones belonging to the associated $\mathrm{GO}$ categories.

It is believed that aganglionosis that characterizes Hirschsprung can arise from: (1) reduced size of the stem/progenitor cell pool; (2) loss of stem cell potency leading to premature differentiation of progenitors; (3) reduced cell survival/ increased cell death; (4) intrinsic, cell-autonomous migration defect of the ENCC; (5) abnormal gut microenvironment; and (6) abnormal interaction of enteric neural crest cells with the gut microenvironment. However, factors that affect the ENCC motility/proliferation/differentiation, and thus probably implicated in HSCR, are not clearly known, as are not how the intrinsic cell migration ability or the NCC-gut microenvironment interaction is controlled at the cellular and molecular level. The PBA here conducted on affected patients, not only confirms the most accepted theory for the biological processes implicated in HSCR, but also provides an important initial hypothesis to select candidate genes for further evaluation in the context of the disease. In this sense, and to reinforce the power of the proposed approach, a significant association to the disease could be confirmed for 4 genes after the evaluation of 68 candidate genes chosen on the basis of their inclusion in the GOs associated to the disease.

Moreover, expression of those genes was demonstrated through RT-PCR and immunohistochemistry analyses in human postnatal gut tissue, obtained from patients with different clinical conditions. Today is widely accepted that neurogenesis takes place in human postnatal ENS, a process which mimics the embryonic events during the formation of the ENS. Due to the limitations of working with human embryonic gut tissue, a postnatal gut context is a powerful tool to identify new genes implicated in the development of ENS, and therefore our results would support again the implication of the tested genes in Hirschsprung disease.

In fact, some of the validated genes had been already related to HSCR either directly or indirectly. That is the case of RASGEF1A gene, whose 3 SNPs selected for validation resulted to be all significantly over-transmitted to affected patients. This is not surprising, since $R A S$ GEF1A is located around $65.5 \mathrm{~Kb}$ upstream RET, and previous TDT studies had already showed statistically significant disease associations spanning a region immediately $5^{\prime}$ of $R E T$ through to this gene [16] reflecting the 


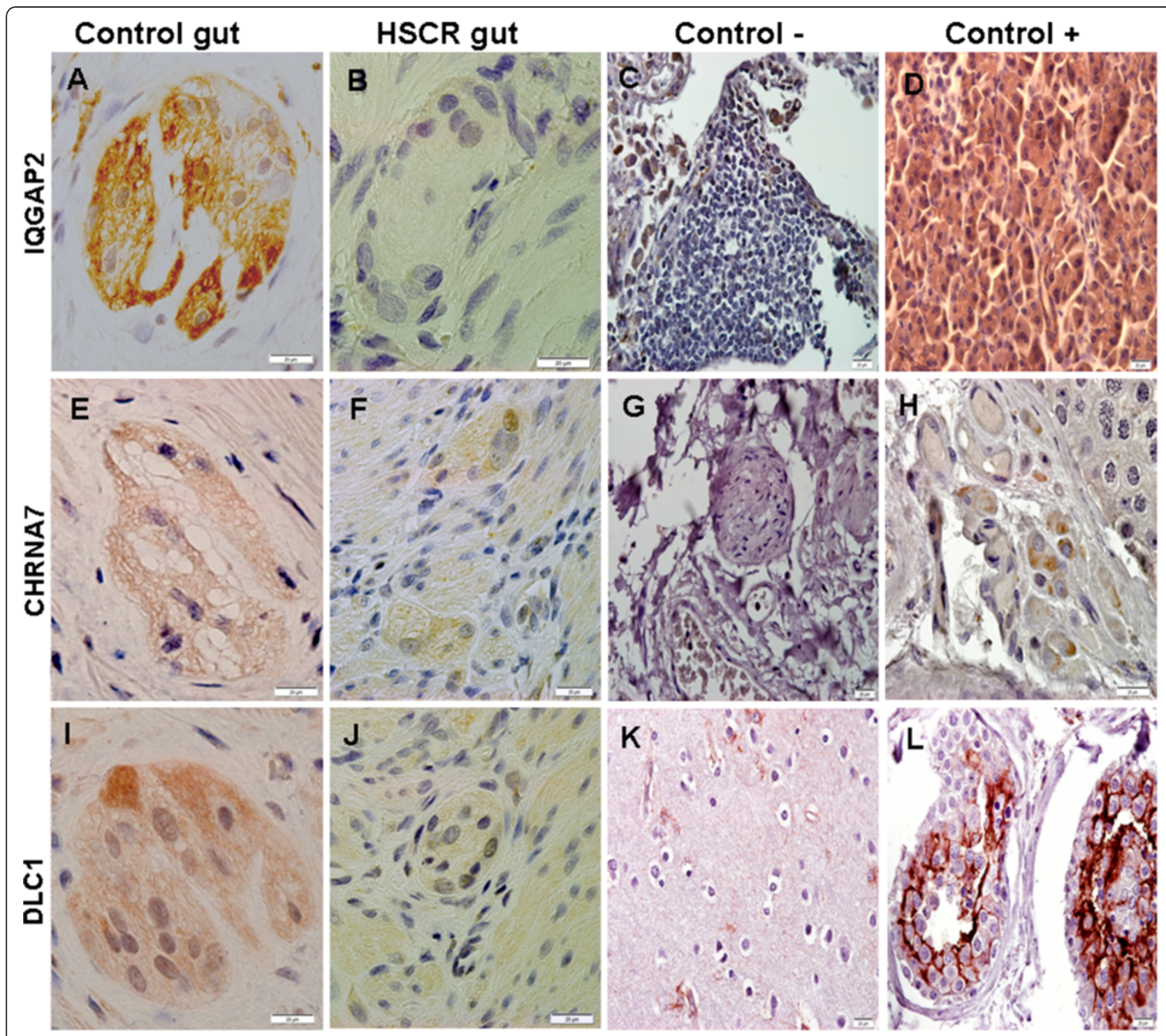

Figure 3 Expression analysis by immunohistochemistry of IQGAP2 (A-D), CHRNA7 (E-H) and DLC1 (I-L) in human tissues: normal gut $(A, E, I)$, HSCR gut $(B, F, J)$, lung $(C, G)$, pancreas $(D)$, testis $(H, L)$ and cerebral cortex (K). Both normal and HSCR guts represent myenteric plexuses. Scale bars: $20 \mu \mathrm{m}$.

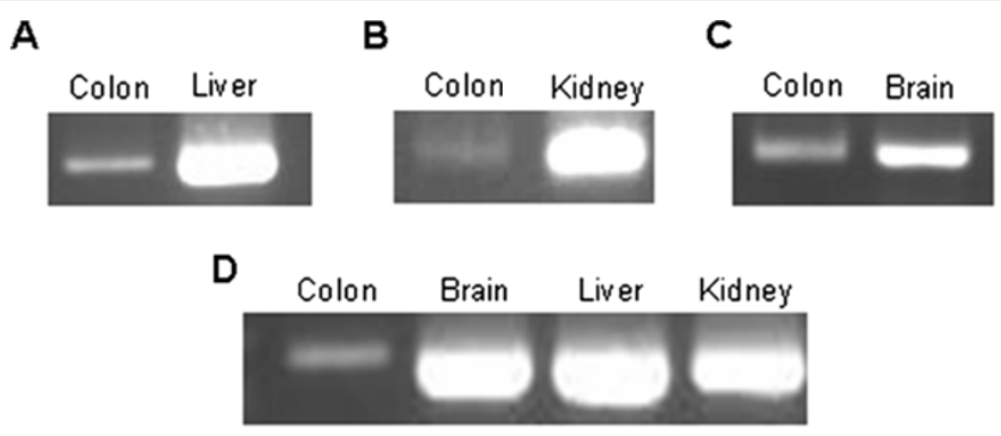

Figure 4 Expression analysis by RT-PCR of IQGAP2 (A), CHRNA7 (B) and DLC1 (C) in human gut tissues. cDNAs of kidney, brain and liver have been used as positive controls for RT-PCR. PCR of B-actin was performed with the different CDNAs used in the study (D). 


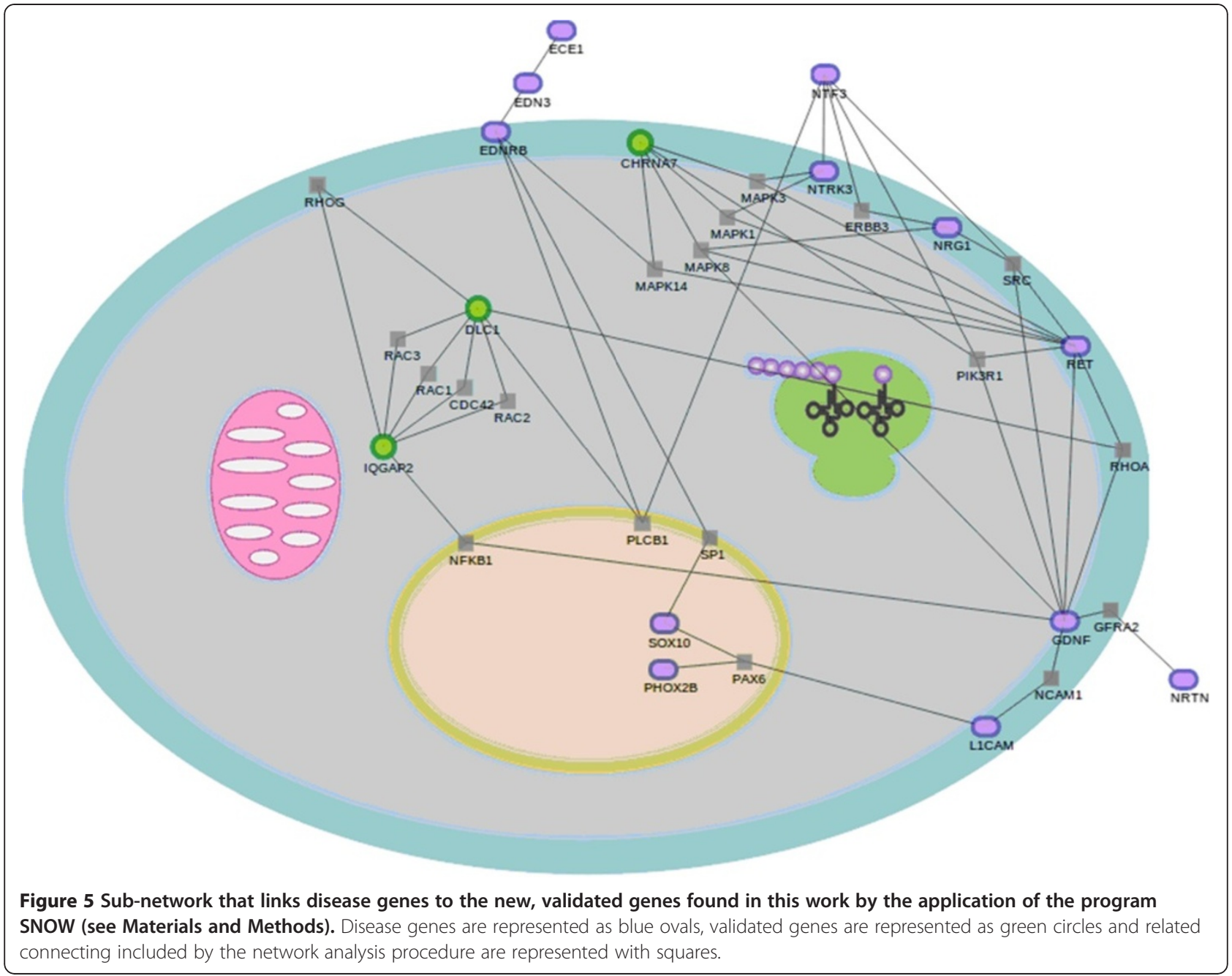

high background linkage disequilibrium in this region. RasGEF1A acts as very specific guanine nucleotide exchange factor for Rap2, a member of the Rap subfamily of Ras-like G-proteins implicated in the regulation of cell adhesion, the establishment of cell morphology, and the modulation of synapses in neurons [51]. Although association tests have excluded the occurrence of a common mutation at RASGEF1A in HSCR, the possibility remains that this gene might carry relevant rare mutations related to HSCR. Importantly, RASGEF1A has been reported to be highly expressed in early embryonic development, at a stage coincident with peak RET expression and colonization of the gut by neural crest-derived neuronal precursors, which would fit with a potential role in enteric neural crest migration [16]. Regarding CHRNA7, it encodes a nicotinic acetylcholine receptor implicated in synaptic transmission regulated by NRG1 among others [52]. Very interestingly, NRG1 has been associated to the disease through both common $[17,53]$ and rare variants [6,54]. In addition, NRG1 SNPs can modulate significantly CHRNA7 expression [52] and for this reason it would be necessary to evaluate the possibility that NRG1 SNPs associated to HSCR act in combination with CHRNA7 leading to a susceptibility for the manifestation of HSCR phenotype.

On the other hand, the remaining significant genes have major functions in early embryogenesis or ENS development, which makes conceivable that, although not previously related to HSCR, they might play a role in its pathogenesis. DLC1 is essential for embryonic development affecting to neural tube development [55] modulating the cytoskeleton and producing morphological changes [56]. IQGAP2 function is still unclear, although studies in with morpholinos $X$. laevis showed that it regulates cell-cell adhesion during early development $[57,58]$. According to the network analysis, IQGAP2 is strongly connected to $D L C 1$ through five simultaneous connections mediated by the genes $R A C 1, R A C 2, R A C 3$, RHOG and CDC42. It is also connected to GDNF (already associated to HSCR) through the NFKB1 gene. 
So, despite its still undefined functional role, the experimental evidences of protein interactions firmly link IQGAP2 to the disease.

In addition valuable information has been also obtained from the network analysis, since it has let us to link the newly identified genes to ones already known to be involved in Hirschsprung. This supports of the associations found and reinforces the role of the new genes in the disease, even though when real network associated to Hirschsprung is probably underestimated because of the lack of information on some gene interactions.

In summary, here we report a number of new candidate genes for HSCR. They need further investigation to elucidate their role in the disease and must be also validated in other populations to discern if their effect in HSCR is universal or is restricted to the Spanish population. Nevertheless, our most important conclusion is that this comprehensive profile of GO terms has demonstrated to be a useful resource for developmental, biochemical and genetic studies. Our report indicate that this approach can help to identify candidate genes for human disease susceptibility loci. These findings could be of especial importance in the field of rare diseases, where large cohorts are often unavailable. In that scenario, the lower sample size requirements make of this approach a suitable and efficient alternative to markerbased analyses.

Beyond technical considerations on the advantages of using GO modules in the analysis of genotype data, the biological pathways highlighted by our study provide insights into the complex nature of HSCR, opens new opportunities for validation of new disease genes and may help in the definition of relatively tractable targets for therapeutic intervention.

\section{Availability of supporting data}

The data sets supporting the results of this article are available in the GEO repository, [http://0-www.ncbi.nlm.nih. gov.opac.acc.msmc.edu/geo/query/acc.cgi?acc=GSE33732].

\section{Additional files}

Additional file 1: Figure S1. Schema of the procedure followed to discover new loci associations. A) The application of a conventional SNPbased TDT on the data resulting from the GWAS in the $53 \mathrm{HSCR}$ trios produces a list of SNPs ranked by p-value. B) SNPs are mapped to genes and genes are thus ranked according to the best (lowest) p-value of the corresponding SNPS. Extragenic SNPS are not used under this approach. C) PBA test produces a list of GO terms significantly over-represented among the genes with best $p$-values and thus, associated to the disease (see text). D) Genes belonging to the significant GO terms and with a nominal (unadjusted) $p$-value $<0.05$ in the step B are used as First Stage candidate genes. E) New SNPs are selected for these genes and genotyped on an independent cohort. F) The application of an association test produces a list of SNPS that are ranked by FDR-adjusted p-values. G) SNPs with adjusted p-values lower than 0.05 are considered markers for the corresponding loci significantly associated to the disease.
H) Network enrichment analysis is conducted to check whether the genes selected are significantly linked among them and to other already known disease genes.

Additional file 2: Table S1. Genes included in the study and SNPS selected to evaluate them in our HSCR cohort.

\section{Abbreviations}

ENCC: Enteric Neural Crest Cells; ENS: Enteric Nervous System; FDR: False Discovery Rate; GO: Gene Ontology; GWAS: Genome-Wide Association Study; HSCR: Hirschprung's; OMIM: On-line Mendelian Inheritance in Man; PBA: Pathway-Based Analysis; SNP: Single Nucleotide Polymosphism; TCA: Total Colonic Aganglionosis.

\section{Competing interests}

The authors declare that they have no competing interests.

\section{Authors' contributions}

RMF, SB, GA and JD drafted the manuscript. RMF, RN-T, BL-T, AT and MVE-R carried out the molecular and expression analyses. MB and MM carried out the two-stage analysis. DM supervised the statistical aspects of the work. LGA carried out the network analysis. IM developed part of the software used in the analysis. JD conceived and coordinated the data analysis. SB conceived the study and coordinated all the laboratory tasks. All authors read and approved the final manuscript.

\section{Acknowledgements}

This work was supported by the Instituto de Salud Carlos III (ISCIII), Spanish Ministry of Economy and Competitiveness, Spain (PI1001290); Spanish Ministry of Economy and Competitiveness (BIO2008-04212), GVA-FEDER (PROMETEO/2010/001) and Consejeria de Innovación Ciencia y Empresa de la Junta de Andalucia (CTS-7447). The CIBER de Enfermedades Raras is an initiative of the ISCIII, Spanish Ministry of Economy and Competitiveness. LGA and MVE-R are supported by fellowships PFIS FI10/00020 and FI11/00533 from ISCIII respectively.

\section{Author details}

${ }^{1}$ Department of Genetics, Reproduction and Fetal Medicine, Institute of Biomedicine of Seville (IBIS, University Hospital Virgen del Rocío/CSIC/ University of Seville, Seville, Spain. ${ }^{2}$ Centre for Biomedical Network Research on Rare Diseases (CIBERER), Barcelona, Spain. ${ }^{3}$ Department of Bioinformatics, Research Centre Príncipe Felipe, Valencia, Spain. ${ }^{4}$ Functional Genomics Node (INB), Research Centre Príncipe Felipe, Valencia, Spain.

Received: 17 April 2012 Accepted: 19 December 2012 Published: 28 December 2012

\section{References}

1. Amiel J, Sproat-Emison E, Garcia-Barcelo M, Lantieri F, Burzynski G, Borrego S, Pelet A, Arnold S, Miao X, Griseri P, et al: Hirschsprung disease, associated syndromes and genetics: a review. J Med Genet 2008, 45:1-14.

2. Emison ES, Garcia-Barcelo M, Grice EA, Lantieri F, Amiel J, Burzynski G, Fernandez RM, Hao L, Kashuk C, West K, et al: Differential contributions of rare and common, coding and noncoding Ret mutations to multifactorial Hirschsprung disease liability. Am J Hum Genet 2010, 87:60-74.

3. Ruiz-Ferrer M, Fernandez RM, Antinolo G, Lopez-Alonso M, Borrego S: NTF3 , a gene involved in the enteric nervous system development, as a candidate gene for Hirschsprung disease. J Pediatr Surg 2008, 43:1308-1311

4. Fernandez RM, Sanchez-Mejias A, Mena MD, Ruiz-Ferrer M, Lopez-Alonso M, Antinolo G, Borrego S: A novel point variant in NTRK3, R645C, suggests a role of this gene in the pathogenesis of Hirschsprung disease. Ann Hum Genet 2009, 73:19-25.

5. Ruiz-Ferrer M, Torroglosa A, Luzon-Toro B, Fernandez RM, Antinolo G, Mulligan LM, Borrego S: Novel mutations at RET ligand genes preventing receptor activation are associated to Hirschsprung's disease. J Mol Med (Berl) 2011, 89:471-480

6. Tang CS, Ngan ES, Tang WK, So MT, Cheng G, Miao XP, Leon TY, Leung BM, Hui KJ, Lui VH, et al: Mutations in the NRG1 gene are associated with Hirschsprung disease. Hum Genet 2011, 131:67-76. 
7. Ruiz-Ferrer M, Torroglosa A, Núñez-Torres R, de Agustín JC, Antiñolo G, Borrego S: Expression of PROKR1 and PROKR2 in human enteric neural precursor cells and identification of sequence variants suggest a role in HSCR. PLoS One 2011, 6:e23475

8. Jiang Q, Turner T, Sosa MX, Rakha A, Arnold S, Chakravarti A: Rapid and efficient human mutation detection using a bench-top next-generation DNA sequencer. Hum Mutat 2012, 33:281-289.

9. Bolk S, Pelet A, Hofstra RM, Angrist M, Salomon R, Croaker D, Buys CH, Lyonnet S, Chakravarti A: A human model for multigenic inheritance: phenotypic expression in Hirschsprung disease requires both the RET gene and a new 9q31 locus. Proc Natl Acad Sci USA 2000, 97:268-273.

10. Gabriel SB, Salomon R, Pelet A, Angrist M, Amiel J, Fornage M, Attie-Bitach T, Olson JM, Hofstra R, Buys C, et al: Segregation at three loci explains familial and population risk in Hirschsprung disease. Nat Genet 2002, 31:89-93.

11. Garcia-Barcelo MM, Fong PY, Tang CS, Miao XP, So MT, Yuan ZW, Li L, Guo WH, Liu L, Wang B, et al: Mapping of a Hirschsprung's disease locus in 3p21. Eur J Hum Genet 2008, 16:833-840.

12. Carrasquillo MM, McCallion AS, Puffenberger EG, Kashuk CS, Nouri N, Chakravarti A: Genome-wide association study and mouse model identify interaction between RET and EDNRB pathways in Hirschsprung disease. Nat Genet 2002, 32:237-244.

13. Lin S, Chakravarti A, Cutler DJ: Exhaustive allelic transmission disequilibrium tests as a new approach to genome-wide association studies. Nat Genet 2004, 36:1181-1188.

14. Brooks AS, Leegwater PA, Burzynski GM, Willems PJ, de Graaf B, van Langen I, Heutink P, Oostra BA, Hofstra RM, Bertoli-Avella AM: A novel susceptibility locus for Hirschsprung's disease maps to 4q31.3-q32.3. J Med Genet 2006, 43:e35.

15. Borrego S, Wright FA, Fernández RM, Williams N, López-Alonso M, Davuluri R, Antiñolo G, Eng C: A founding locus within the RET proto-oncogene may account for a large proportion of apparently sporadic Hirschsprung disease and a subset of cases of sporadic medullary thyroid carcinoma. Am J Hum Genet 2003, 72:88-100.

16. Emison ES, McCallion AS, Kashuk CS, Bush RT, Grice E, Lin S, Portnoy ME, Cutler DJ, Green ED, Chakravarti A: A common sex-dependent mutation in a RET enhancer underlies Hirschsprung disease risk. Nature 2005, 434:857-863.

17. Garcia-Barcelo MM, Tang CS, Ngan ES, Lui VC, Chen Y, So MT, Leon TY, Miao XP, Shum CK, Liu FQ, et al: Genome-wide association study identifies NRG1 as a susceptibility locus for Hirschsprung's disease. Proc Natl Acad Sci USA 2009, 106:2694-2699.

18. Wang K, Li M, Hakonarson H: Analysing biological pathways in genomewide association studies. Nat Rev Genet 2010, 11:843-854.

19. Aulchenko YS, Ripatti S, Lindqvist I, Boomsma D, Heid IM, Pramstaller PP, Penninx BW, Janssens AC, Wilson JF, Spector T, et al: Loci influencing lipid levels and coronary heart disease risk in 16 European population cohorts. Nat Genet 2009, 41:47-55.

20. Askland K, Read C, Moore J: Pathways-based analyses of whole-genome association study data in bipolar disorder reveal genes mediating ion channel activity and synaptic neurotransmission. Hum Genet 2009, 125:63-79.

21. Torkamani A, Topol EJ, Schork NJ: Pathway analysis of seven common diseases assessed by genome-wide association. Genomics 2008, 92:265-272

22. Vidal M, Cusick ME, Barabasi AL: Interactome networks and human disease. Cell 2011, 144:986-998

23. Barabasi AL, Gulbahce N, Loscalzo J: Network medicine: a network-based approach to human disease. Nat Rev Genet 2011, 12:56-68.

24. Baranzini SE: The genetics of autoimmune diseases: a networked perspective. Curr Opin Immunol 2009, 21:596-605.

25. Lim J, Hao T, Shaw C, Patel AJ, Szabo G, Rual JF, Fisk CJ, Li N, Smolyar A, Hill $D E$, et al: A protein-protein interaction network for human inherited ataxias and disorders of Purkinje cell degeneration. Cell 2006, 125:801-814.

26. Goehler H, Lalowski M, Stelzl U, Waelter S, Stroedicke M, Worm U, Droege A, Lindenberg KS, Knoblich M, Haenig C, et al: A protein interaction network links GIT1, an enhancer of huntingtin aggregation, to Huntington's disease. Mol Cell 2004, 15:853-865.

27. Camargo LM, Collura V, Rain JC, Mizuguchi K, Hermjakob H, Kerrien S, Bonnert TP, Whiting PJ, Brandon NJ: Disrupted in Schizophrenia 1
Interactome: evidence for the close connectivity of risk genes and a potential synaptic basis for schizophrenia. Mol Psychiatry 2007, 12:74-86.

28. Soler-Lopez M, Zanzoni A, Lluis R, Stelzl U, Aloy P: Interactome mapping suggests new mechanistic details underlying Alzheimer's disease. Genome Res 2011, 21:364-376.

29. Purcell S, Neale B, Todd-Brown K, Thomas L, Ferreira MA, Bender D, Maller J, Sklar P, de Bakker PI, Daly MJ, Sham PC: PLINK: a tool set for wholegenome association and population-based linkage analyses. Am J Hum Genet 2007, 81:559-575.

30. Wang K, Li M, Bucan M: Pathway-based approaches for analysis of genomewide association studies. Am J Hum Genet 2007, 81:1278-1283.

31. Medina I, Montaner D, Bonifaci N, Pujana MA, Carbonell J, Tarraga J, AlShahrour F, Dopazo J: Gene set-based analysis of polymorphisms: finding pathways or biological processes associated to traits in genome-wide association studies. Nucleic Acids Res 2009, 37:W340-W344

32. Al-Shahrour F, Carbonell J, Minguez P, Goetz S, Conesa A, Tarraga J, Medina I, Alloza E, Montaner D, Dopazo J: Babelomics: advanced functional profiling of transcriptomics, proteomics and genomics experiments. Nucleic Acids Res 2008, 36:W341-W346.

33. Medina I, Carbonell J, Pulido L, Madeira SC, Goetz S, Conesa A, Tarraga J, Pascual-Montano A, Nogales-Cadenas R, Santoyo J, et al: Babelomics: an integrative platform for the analysis of transcriptomics, proteomics and genomic data with advanced functional profiling. Nucleic Acids Res 2010, 38(Suppl):W210-W213.

34. Ashburner M, Ball CA, Blake JA, Botstein D, Butler H, Cherry JM, Davis AP, Dolinski K, Dwight SS, Eppig JT, et al: Gene ontology: tool for the unification of biology. The gene ontology consortium. Nat Genet 2000, 25:25-29.

35. Benjamini $Y$, Hochberg $Y$ : Controlling the false discovery rate: a practical and powerful approach to multiple testing. J Roy Stat Soc B 1995, 57:289-300

36. Hirschhorn JN, Daly MJ: Genome-wide association studies for common diseases and complex traits. Nat Rev Genet 2005, 6:95-108.

37. Zaghloul NA, Katsanis N: Functional modules, mutational load and human genetic disease. Trends Genet 2010, 26:168-176.

38. Todd JA: Statistical false positive or true disease pathway? Nat Genet 2006, 38:731-733.

39. Heller R, Manduchi E, Grant GR, Ewens WJ: A flexible two-stage procedure for identifying gene sets that are differentially expressed. Bioinformatics 2009, 25:1019-1025.

40. Conde L, Vaquerizas JM, Dopazo H, Arbiza L, Reumers J, Rousseau F, Schymkowitz J, Dopazo J: PupaSuite: finding functional single nucleotide polymorphisms for large-scale genotyping purposes. Nucleic Acids Res 2006, 34:W621-W625.

41. Ideker T, Sharan R: Protein networks in disease. Genome Res 2008, 18:644-652.

42. Minguez P, Dopazo J: Assessing the biological significance of gene expression signatures and co-expression modules by studying their network properties. PLOS One 2011, 6:e17474.

43. Minguez P, Gotz S, Montaner D, Al-Shahrour F, Dopazo J: SNOW, a webbased tool for the statistical analysis of protein-protein interaction networks. Nucleic Acids Res 2009, 37:W109-W114.

44. D'Eustachio P: Reactome knowledgebase of human biological pathways and processes. Methods Mol Biol 2011, 694:49-61.

45. Laranjeira C, Pachnis V: Enteric nervous system development: Recent progress and future challenges. Auton Neurosci 2009, 151:61-69.

46. Schadt EE: Molecular networks as sensors and drivers of common human diseases. Nature 2009, 461:218-223.

47. Dopazo J: Formulating and testing hypotheses in functional genomics. Artif Intell Med 2009, 45:97-107.

48. Ideker T, Dutkowski J, Hood L: Boosting signal-to-noise in complex biology: prior knowledge is power. Cell 2011, 144:860-863.

49. Stenson PD, Mort M, Ball EV, Howells K, Phillips AD, Thomas NS, Cooper DN: The Human Gene Mutation Database: 2008 update. Genome Med 2009, 1:13.

50. Cooper DN, Chen JM, Ball EV, Howells K, Mort M, Phillips AD, Chuzhanova N, Krawczak M, Kehrer-Sawatzki H, Stenson PD: Genes, mutations, and human inherited disease at the dawn of the age of personalized genomics. Hum Mutat 2010, 31:631-655.

51. Yaman E, Gasper R, Koerner C, Wittinghofer A, Tazebay UH: RasGEF1A and RasGEF1B are guanine nucleotide exchange factors that discriminate between Rap GTP-binding proteins and mediate Rap2-specific nucleotide exchange. FEBS J 2009, 276:4607-4616. 
52. Mathew SV, Law AJ, Lipska BK, Davila-Garcia MI, Zamora ED, Mitkus SN, Vakkalanka R, Straub RE, Weinberger DR, Kleinman JE, Hyde TM: Alpha7 nicotinic acetylcholine receptor mRNA expression and binding in postmortem human brain are associated with genetic variation in neuregulin 1. Hum Mol Genet 2007, 16:2921-2932.

53. Phusantisampan T, Sangkhathat S, Phongdara A, Chiengkriwate $P$, Patrapinyokul S, Mahasirimongkol S: Association of genetic polymorphisms in the RET-protooncogene and NRG1 with Hirschsprung disease in Thai patients. J Hum Genet, :. in press.

54. Luzon-Toro B, Torroglosa A, Nuñez-Torres R, Enguix-Riego MV, Fernandez RM, De Agustin JC, Antiñolo G, Borrego S: Comprehensive analysis of NRG1 common and rare variants in Hirschsprung patients. PLOS One. in press.

55. Durkin ME, Avner MR, Huh CG, Yuan BZ, Thorgeirsson SS, Popescu NC: DLC1, a Rho GTPase-activating protein with tumor suppressor function, is essential for embryonic development. FEBS Lett 2005, 579:1191-1196.

56. Liao YC, Lo SH: Deleted in liver cancer-1 (DLC-1): a tumor suppressor not just for liver. Int I Biochem Cell Biol 2008, 40:843-847.

57. Yamashiro S, Abe H, Mabuchi I: IQGAP2 is required for the cadherinmediated cell-to-cell adhesion in Xenopus laevis embryos. Dev Biol 2007, 308:485-493.

58. White CD, Brown MD, Sacks DB: IQGAPs in cancer: a family of scaffold proteins underlying tumorigenesis. FEBS Lett 2009, 583:1817-1824.

doi:10.1186/1750-1172-7-103

Cite this article as: Fernández et al:: Four new loci associations discovered by pathway-based and network analyses of the genomewide variability profile of Hirschsprung's disease. Orphanet Journal of Rare Diseases 2012 7:103.

\section{Submit your next manuscript to BioMed Central and take full advantage of:}

- Convenient online submission

- Thorough peer review

- No space constraints or color figure charges

- Immediate publication on acceptance

- Inclusion in PubMed, CAS, Scopus and Google Scholar

- Research which is freely available for redistribution 